\title{
Efeito de índices técnicos na rentabilidade de propriedades leiteiras participantes do programa "Balde Cheio"
}

\author{
$\underline{\text { Flávio de Moraes }}^{1}, \underline{\text { Marcos Aurélio Lopes }}^{2 *}, \underline{\text { Fábio Raphael Pascoti Bruhn }}{ }^{3}$, $\underline{\text { Afonso Aurélio }}$ \\ $\underline{\text { de Carvalho Peres }}^{4}$, André Luis Ribeiro Lima $^{5}$, Eduardo Mitke Brandão Reis ${ }^{6}$
}

${ }^{I}$ MSc. Médico Veterinário autônomo, Rio de Janeiro, RJ, Brasil.f.demoraes2009@gmail.com

${ }^{2}$ Universidade Federal de Lavras, Departamento de Medicina Veterinária, Lavras, MG, Brasil. malopes@dmv.ufla.br.

${ }^{3}$ Universidade Federal de Pelotas, Faculdade de Medicina Veterinária, Capão do Leão, RS, Brasil.fabio rpb@yahoo.com.br

${ }^{4}$ Universidade Federal Fluminense, Departamento de Engenharia de Agronegócios, Volta Redonda, RJ, Brasil. lelo@metal.eeimvr.uff.br

${ }^{5}$ Universidade Federal de Lavras, Departamento de Economia e Administração, Lavras, MG, Brasil. andre.lima@dae.ufla.br

${ }^{6}$ Universidade Federal do Acre, Centro de Ciências Biológicas e da Natureza, Rio Branco, AC, Brasil. edumitke@hotmail.com

*Autor para correspondência

RESUMO. Objetivou-se analisar o efeito de alguns índices técnicos e gerenciais na rentabilidade de 20 unidades demonstrativas (UD) participantes do programa "Balde Cheio", no estado do Rio de Janeiro, no período de janeiro a dezembro de 2011. Realizou-se a análise de rentabilidade considerando a margem bruta, a margem líquida, o resultado (lucro ou prejuízo), a rentabilidade e a lucratividade como indicadores de eficiência econômica. Para descrever os indicadores de rentabilidade, bem como os índices técnicos e gerenciais, foi utilizada a estatística descritiva por meio da média, desvio padrão, valor mínimo e valor máximo, calculadas utilizando-se o software estatístico SPSS 18.0. Os índices técnicos e gerenciais influenciaram na lucratividade e na rentabilidade nas propriedades estudadas. Os índices zootécnicos, porcentagem de vaca em lactação, vacas no rebanho e vacas em lactação no rebanho, produtividade animal/ha/ano e quantidade de vacas em lactação/ha foram considerados bons; porém, perceberam-se que embora as margens bruta e líquida sejam positivas, os produtores a longo prazo estão se descapitalizando, pois o resultado foi negativo.

Palavras chave: Bovinocultura de leite, bovinos, gerenciamento, índices zootécnicos e gerenciais, programa Balde Cheio.

\section{Effect of technical indices on the profitability in dairy properties participating of "Balde Cheio" program}

\begin{abstract}
The scope of this research was to analyze the effect of some technical and managerial levels in the profitability of 20 demonstration units (UD) participants of the program "Balde Cheio", in the State of Rio de Janeiro, from January to December in 2011. The profitability analysis considering the gross margin, the net margin, the result (profit or loss), profitability and rentability as indicators of economic efficiency. To describe the profitability indicators, as well as the technical and managerial levels, descriptive statistics was used through the mean, standard deviation, minimum and maximum value, calculated using the statistical software SPSS 18.0. The technical and managerial levels influenced on profitability and rentability in the properties studied. The zootechnical rates percentage of lactating cow, cows in the herd and lactating cows in the herd, animal productivity/ha/year and amount of lactating cows/ha were considered good; However, noted that although gross and net margins are positive, in the long-term producers are in the process of decapitalising, because the result was negative.
\end{abstract}

Keywords: "Balde Cheio" program cost of production, dairy cattle, management technical indices. 


\section{Introdução}

A bovinocultura de leite é uma atividade de grande importância para o desenvolvimento econômico brasileiro e um dos produtos mais importantes da agropecuária; além de admitir a fixação do homem no campo, amortizando as pressões sociais nas áreas urbanas e colaborar para minimização do desemprego e da exclusão social (Gonçalves et al., 2014, FAO, 2015). No entanto, a produção leiteira no país, ainda, é caracterizada por grande heterogeneidade, tanto nas técnicas de produção, quanto no rebanho e perfil dos produtores (Siqueira et al., 2010, ANUALPEC, 2015).

O programa "Balde Cheio" desenvolvido pelo Centro de Pesquisa de Pecuária do Sudeste (CPPSE), da Empresa Brasileira de Pesquisa Agropecuária (EMBRAPA), em São Carlos, SP, surgiu da necessidade de atender os produtores de leite ineficientes, capacitando profissionais da extensão rural e produtores rurais, promovendo a troca de informações sobre as tecnologias aplicadas e monitorando os impactos ambientais, econômicos e sociais nos sistemas de produção (Borges et al., 2011).

A lucratividade da atividade pecuária pode ser avaliada pelos índices técnicos, uma vez que eles estão relacionados à produção e, consequentemente, aos lucros do produtor. Assim, produtores e técnicos devem estar atentos para calcular os índices e identificar aqueles que afetam a rentabilidade da atividade, maximizando a produção e minimizando os custos (Lopes et al., 2009).

Vários pesquisadores têm se preocupado em estimar o custo de produção e estudar a viabilidade econômica da produção de leite (Carlotto et al., 2011, Silveira et al., 2011, Godinho \& Carvalho, 2013). Entretanto, raros estudaram o efeito de índices técnicos e gerenciais na rentabilidade da produção leiteira. Considerando a importância do programa "Balde Cheio" e do tema para o país e para o estado do Rio de Janeiro, bem como a escassez de trabalhos científicos, objetivou-se estudar o efeito de alguns índices técnicos e gerenciais na rentabilidade de 20 Unidades Demonstrativas (UD), participantes do programa "Balde Cheio".

\section{Material e Métodos}

Os dados analisados foram provenientes de 20 sistemas de produção de leite, denominados de
Unidades Demonstrativas (UDs), participantes do programa "Balde Cheio", localizados no estado do Rio de Janeiro, no ano de 2011. O município de Natividade apresentou duas UDs; Valença, Carmo e Campos dos Goytacazes, três cada; e Quatis, Barra Mansa, Barra do Piraí, Paraíba do Sul, Santa Maria Madalena, Aperibé, Conceição de Macabu, Itaperuna e Varre-Sai apresentou apenas uma. Essas UDs foram escolhidas utilizando-se amostragem não probabilística por julgamento, levando-se em consideração os seguintes critérios: disponibilidade e qualidade de dados zootécnicos e financeiros; consentimento e interesse do pecuarista na realização da pesquisa; facilidade de acesso por parte do pesquisador às fontes de evidências. Os dados foram anotados, pelos produtores, em cadernetas de campo ao longo do ano e colhidos a cada visita mensal pelo técnico responsável pela UD.

Foram usados como indicadores de rentabilidade, a margem bruta (receita menos custo operacional efetivo), margem líquida (receita menos custo operacional total) (Matsunaga et al., 1976) e resultado (receita menos custo total) (Lopes et al., 2007).

Foi estimada a lucratividade 1, calculada como sendo o resultado dividido pela receita total, multiplicado por 100 (Lucratividade 1 (\%) $=$ Resultado/Receita total x 100); e a rentabilidade 1 , obtida pela divisão do resultado pelo total imobilizado mais o custo operacional efetivo, multiplicado por 100 (Rentabilidade 1 $(\%)=$ Resultado/(Total imobilizado + Custo operacional efetivo) x 100). Como lucratividade 2, foi considerada a divisão da margem líquida pela receita total, multiplicado por 100 (Lucratividade $2(\%)=$ Margem líquida/Receita total x 100); e, como rentabilidade 2, foi considerada a divisão da margem líquida pelo total imobilizado mais o custo operacional efetivo, multiplicado por 100 (Rentabilidade 2 $(\%)=$ Margem líquida/(Total imobilizado + Custo operacional efetivo) x 100) (Lopes et al., 2011).

$\mathrm{Na}$ realização do inventário completo dos bens, o valor e a vida útil em relação ao tempo de aquisição de cada ativo foram apurados e posteriormente, agrupados em categorias preestabelecidas: benfeitorias, máquinas, veículos, equipamentos, implementos, ferramentas, rebanho e móveis. Nas situações nas quais os pecuaristas não dispunham de informações referentes ao valor e data de 
aquisição, para a estimativa dos valores atualizados, bem como da vida útil restante, foram adotados os critérios propostos por Lopes et al. (2004).

Para descrever os indicadores margem bruta, margem líquida, resultado, rentabilidade e lucratividade, bem como os índices técnicos e gerenciais foi utilizada a estatística descritiva por meio da média, desvio padrão, valor mínimo e valor máximo, calculadas utilizando o software estatístico SPSS 18.0.

\section{Resultados e Discussão}

Os índices técnicos e gerenciais de 20 Unidades Demonstrativas participantes do programa "Balde Cheio", no estado do Rio de Janeiro, no período de janeiro a dezembro de 2011 podem ser observados na tabela 1 e, um resumo da análise de rentabilidade na tabela 2 . A razão matriz/mão de obra também supera os 14,96 encontrados por Lopes et al. (2004) e os 12,17 relatados por Lopes et al. (2006). Em ambos os estudos citados, a atividade leiteira não foi viável economicamente em longo prazo, pois quando se analisa pelo indicador de eficiência econômica (receita bruta menos custo total) os resultados foram insatisfatórios, evidenciando que a atividade leiteira não conseguiu remunerar o capital, sendo observados resultados semelhantes aos encontrados nesse estudo.

Tabela 1. Estatísticas descritivas dos índices técnicos e gerenciais de 20 Unidades Demonstrativas do programa "Balde Cheio", no estado do Rio de Janeiro (período de janeiro a dezembro de 2011)

\begin{tabular}{lccccc}
\hline \multicolumn{1}{c}{ Especificação } & Unidade & Média & DP & Mínimo & Máximo \\
\hline Relação matrizes / mão de obra & un & 16,43 & 9,93 & 4,83 & 37,17 \\
Relação de quantidade animais do rebanho /mão de obra & un & 31,83 & 30,39 & 6,67 & 131,67 \\
Animais / área para produção & UA/ha & 5,29 & 4,40 & 1,52 & 17,79 \\
Vacas em lactação & $\%$ & 75,13 & 10,01 & 52,98 & 88,75 \\
Vacas no rebanho & $\%$ & 63,43 & 17,14 & 27,34 & 100,00 \\
Vacas em lactação no rebanho & $\%$ & 48,11 & 16,21 & 19,43 & 88,75 \\
Produção animal / dia & kg & 11,76 & 3,16 & 5,42 & 16,47 \\
Produtividade animal / ha / ano & $\mathrm{kg}$ & $10.016,92$ & $15.633,05$ & 591,03 & $71.126,00$ \\
Ponto de equilíbrio / kg / leite / dia & $\mathrm{kg}$ & $2.123,97$ & $5.462,05$ & 78,44 & $20.761,78$ \\
Ponto de equilíbrio operacional / dia & $\mathrm{kg}$ & 762,99 & $1.967,47$ & 36,04 & $7.438,90$ \\
Quantidade de leite produzido total & $\mathrm{kg}$ & $75.879,70$ & $52.629,49$ & $24.359,00$ & $228.118,00$ \\
Quantidade de leite produzido / dia & $\mathrm{kg}$ & 207,89 & 144,19 & 66,74 & 624,98 \\
Quantidade de vacas em lactação / ha & un & 2,88 & 3,07 & 0,00 & 13,33 \\
Produção de leite / mão de obra & $\mathrm{kg} / \mathrm{serviço}$ & 142,87 & 96,55 & 48,28 & 446,24 \\
Quantidade de mão de obra (contratada ou familiar) & un & 1,55 & 0,69 & 1,00 & 3,00 \\
Mão de obra familiar / COT & $\%$ & 20,06 & 14,46 & 0,00 & 45,88 \\
COE / COT & $\%$ & 68,98 & 13,71 & 45,16 & 91,77 \\
Depreciação / COT & $\%$ & 10,96 & 2,36 & 7,83 & 15,61 \\
\hline DP = desvio padrão. & & & & &
\end{tabular}

A média de vacas em lactação (Tabela 1) foi superior a 50\% encontrada no estudo de Sousa et al. (2011) e aos $58 \%$ observados no estudo de Lopes et al. (2010). Observou-se melhor eficiência reprodutiva nas UDs deste estudo, cujo valor médio foi bem próximo de $83,3 \%$ de vacas em lactação, considerados ideais pelo estudo realizado pela Federação da Agricultura, Pecuária e Pesca do Estado de Minas Gerais, em 2006 (FAEMG, 2006).
Quanto à produção diária por animal, o valor médio encontrado (Tabela 1) está abaixo dos 15,1 litros/vaca em lactação/dia encontrado por Lopes et al. (2007). Esses pesquisadores observaram que a obtenção de maiores níveis de produção é condição necessária para um desempenho econômico eficiente.

$\mathrm{O}$ valor médio de produtividade em animal/ha/ano (Tabela 1) foi superior aos 2.614 $\mathrm{kg}$ encontrados por Lopes et al. (2004) e aos $1.188 \mathrm{~kg} / \mathrm{ha} /$ ano relatados no estudo da FAEMG 
(FAEMG, 2006). O valor máximo se destacou com a produção de $71.126 \mathrm{~kg}$ de leite e 13,3 vacas em lactação/ha, valores semelhantes a esses ainda não foram mencionados na literatura científica.

Os pontos de equilíbrio total e operacional (Tabela 1) das UDs foram de 2.124 e $763 \mathrm{~kg}$ de leite por dia, respectivamente, enquanto a produção média diária foi de $207,9 \mathrm{~kg}$ de leite. Tal fato demonstrou à necessidade de se aumentar a escala de produção nas propriedades assistidas. Segundo Lopes et al. (2006), situações como essa evidenciaram que muitos esforços gerenciais e até mesmo tecnológicos devam ser feitos objetivando aumentar as médias diárias, sem, contudo aumentar o custo variável médio, que uma vez majorado, aumentará ainda mais o ponto de equilíbrio. De acordo com esses pesquisadores, uma alternativa é aumentar a eficiência produtiva, ou seja, a produção por matriz, otimizando assim as despesas com mãode-obra, medicamentos, inseminação artificial, impostos fixos, energia e diversas. Tais despesas, aumentando-se a produção por matriz, não serão majoradas.

Quanto à quantidade de vacas em lactação por área (Tabela 1), o valor médio encontrado foi maior do que os 0,80 vacas em lactação/ha obtido por Lopes et al. (2004), do que o 1,39 vacas/ha encontrado por Lopes et al. (2010) e do 1,34 vacas/ha por Fassio et al. (2006). Os dados de produtividade e lotação por área demonstraram que as UDs assistidas pelo programa "Balde Cheio" foram mais eficientes no uso da terra. Essa informação é muito importante, pois deverá influenciar na elaboração de políticas públicas voltadas aos produtores familiares. Como consequência, geraria renda, mais dignidade e resgataria a auto-estima das pessoas; com isso, fixaria o homem no campo, reduzindo o êxodo rural.

A razão produção de leite por mão de obra (Tabela 1) foi superior aos 118 litros/homem encontrado por Oliveira et al. (2007) e 104 litros/homem por Oliveira et al. (2001)e semelhante aos144 litros/homem por Lopes et al. (2004). Quanto à relação da mão de obra familiar pelo COT (Tabela 1), notou-se grande desvio padrão, pois havia UDs que só possuíam mão de obra contratada, justificando os valores mínimo nulos. Embora os índices zootécnicos de porcentagem de vaca em lactação, vacas no rebanho e vacas em lactação no rebanho, produtividade animal/ha/ano e quantidade de vacas em lactação/ha (Tabela 1) tenha sido bons, a lucratividade e rentabilidade foram negativas (Tabela 2).

Tabela 2. Estatísticas descritivas do resumo da análise de rentabilidade da atividade leiteira de 20 Unidades Demonstrativas do programa "Balde Cheio", no estado do Rio de Janeiro (período de janeiro a dezembro de 2011).

\begin{tabular}{lcllll}
\hline Especificação & Unidade & Média & DP & Mínimo & Máximo \\
\hline Margem bruta & $\mathrm{R} \$$ & $28.404,03$ & $17.525,31$ & $11.403,43$ & $77.221,55$ \\
Margem líquida & $\mathrm{R} \$$ & $14.238,40$ & $17.911,83$ & $-2.638,40$ & $68.754,11$ \\
Resultado (lucro ou prejuízo) & $\mathrm{R} \$$ & $-818,63$ & $15.567,47$ & $-26.045,87$ & $45.401,69$ \\
Lucratividade 1 & $\%$ & $-6,94$ & 22,08 & $-74,56$ & 26,46 \\
Rentabilidade 1 & $\%$ & $-1,04$ & 5,37 & $-10,60$ & 9,82 \\
Lucratividade 2 & $\%$ & 15,21 & 13,28 & $-9,12$ & 41,84 \\
Rentabilidade 2 & $\%$ & 5,03 & 4,61 & $-2,66$ & 14,88 \\
\hline
\end{tabular}

DP = Desvio Padrão; Lucratividade 1: Resultado / Receita total; Lucratividade 2: Margem líquida / Receita total; Rentabilidade 1: Resultado / (Custo operacional efetivo + imobilizado total); Rentabilidade 2: Margem líquida / (Custo operacional efetivo + imobilizado total). Fonte: Dados da pesquisa

Analisando a Rentabilidade 2 (Margem líquida / Custo operacional efetivo + total imobilizado), o valor de 5,0\%, embora positivo, é menos atrativo que a remuneração obtida com a caderneta de poupança (7,5\%), no ano de 2011 (Portal Brasil, 2016). Ao analisar a Lucratividade 2 (Margem líquida / Receita total), observou-se ganho de R\$15,21 para cada $\mathrm{R} \$ 100,00$ de receita. Tanto em relação a esse indicador, quanto à Rentabilidade 2, os valores foram maiores em função do fato que em suas estimativas não são consideradas a remuneração da terra, do capital investido, do empresário, dos impostos considerados fixos e da remuneração do capital de giro (Lopes et al., 2009). 
$\mathrm{Na}$ tabela 3 pode ser observada a representatividade de cada item dos recursos disponíveis nas 20 UDs estudadas. Observou-se que $40,4 \%$ do valor estão investidos em terra. Quando não considerado esse bem, os animais são aqueles com maior representatividade $(49,4 \%)$. Devido ao alto valor imobilizado na terra justifica-se, segundo Lopes et al. (2008), a sua otimização, que pode ser atingida pelo aumento da escala de produção das propriedades.

Tabela 3. Estatísticas descritivas da representatividade de cada item do inventário dos recursos disponíveis em 20 Unidades Demonstrativas do programa "Balde Cheio", no estado do Rio de Janeiro (período de janeiro a dezembro de 2011), em \%

\begin{tabular}{lcccc}
\hline Especificação & Média & DP & Mínimo & Máximo \\
\hline Valor do patrimônio em terra & 40,40 & 20,12 & 6,99 & 77,56 \\
Valor do patrimônio sem terra & 59,60 & 20,12 & 22,44 & 93,01 \\
$\quad$ Valor em benfeitorias & 34,84 & 10,73 & 12,32 & 63,10 \\
Valor em equipamentos & 1,51 & 1,12 & 0,33 & 4,66 \\
Valor em ferramentas & 0,12 & 0,06 & 0,02 & 0,23 \\
Valor em implementos & 0,76 & 0,61 & 0,00 & 2,10 \\
Valor em máquinas & 12,38 & 8,18 & 0,00 & 34,07 \\
Valor em animais & 49,41 & 11,23 & 23,66 & 67,20 \\
Valor em semoventes & 0,64 & 0,51 & 0,00 & 1,83 \\
Valor em móveis & 0,34 & 0,16 & 0,07 & 0,70 \\
$\quad$ Valor em veículos & 0,00 & 0,00 & 0,00 & 0,00 \\
Valor do patrimônio em terra $(\mathrm{R} \$)$ & $113.201,02$ & $132.454,86$ & $5.000,00$ & $560.000,00$ \\
Valor do patrimônio sem terra (R\$) & $112.675,40$ & $85.161,58$ & $50.713,00$ & $405.223,00$ \\
\hline DP $=$ d & & & &
\end{tabular}

$\mathrm{DP}=$ desvio padrão.

Para melhorar a lucratividade e rentabilidade, os produtores deveriam melhorar os índices de produção de leite por mão de obra, produção animal por dia e a relação de matrizes por mão de obra.

Os índices técnicos e gerenciais influenciaram nas lucratividades e nas rentabilidades das propriedades estudadas. Os índices zootécnicos e porcentagem vacas no rebanho e vacas em lactação no rebanho, produtividade animal/ha/ano e quantidade de vacas em lactação/ha foram considerados bons, porém percebeu-se que, embora as margens brutas e líquidas sejam positivas, os produtores, em longo prazo, estão se descapitalizado, pois o resultado foi negativo.

\section{Referências Bibliográficas}

ANUALPEC. (2015). Anuário da Pecuária Brasileira, $20^{\mathrm{a}}$ ed. Instituto FNP, São Paulo, SP, Brasil.

Borges, M. S., Guedes, C. A. M. \& Assis, R. L. (2011). Um estudo do "projeto Balde Cheio" como vetor do desenvolvimento sustentável do pequeno produtor de leite. Revista
Brasileira de Agropecuária Sustentável, 1, 151-161.

Carlotto, I., Filippi, J. A. \& Marcello, I. E. (2011). Estudo da viabilidade da produção de leite em uma propriedade familiar rural do município de Francisco Beltrão-PR. Revista de Ciências Empresariais da UNIPAR, 12, 95-109.

FAEMG - Federação da Agricultura, Pecuária e Pesca do Estado de Minas Gerais. (2006). Diagnóstico da pecuária leiteira do Estado de Minas Gerais em 2005: SEBRAE-MG OCEMG E SENAR-AR/MG- Belo Horizonte, $156 p$

FAO. (2015). Statistical Yearbook. Food and Agriculture Organization of the United Nations, Rome, Italy.

Fassio, L. H., Reis, R. P. \& Geraldo, L. G. (2006). Desempenho técnico e econômico da atividade leiteira em Minas Gerais. Ciência e Agrotecnologia, 30, 1154-1161.

Godinho, R. F. \& Carvalho, R. C. R. (2013). Gestão de sistemas de produção de leite. Ciência et Praxis, 2, 77-82. 
Gonçalves, A. C. S., Júnior, L. C. R., Fonseca, M. I., Nadruz, B. V., Bürger, K. P. \& Rossi, G. A. M. (2014). Assistência técnica e extensão rural: um estudo de caso que demonstra sua importância para a melhoria da produção leiteira. Revista Brasileira de Higiene e Sanidade Animal, 8, 47-61.

Lopes, A. D., Oliveira, M. D. S. \& Fonseca, M. I. (2010). Características técnicas das propriedades de baixa escala leiteira observadas na área de abrangência do Escritório de Desenvolvimento Rural de Jaboticabal-SP. Revista Ciência em Extensão, 6, 32-45.

Lopes, M., Cardoso, M., Demeu, F. \& Dias, A. (2008). Composição de rebanhos bovinos leiteiros: levantamento, estimativa do dimensionamento e da evolução. Boletim da Indústria Animal, 65, 163-167.

Lopes, M. A., Dias, A. S., Carvalho, F. M., Lima, A. L. R., Cardoso, M. G. \& Carmo, E. A. (2009). Resultados econômicos de sistemas de produção de leite com diferentes níveis tecnológicos na região de Lavras-MG nos anos 2004 e 2005. Revista Ciência e Agrotecnologia, 33, 252-260.

Lopes, M. A., Lima, A. L. R., Carvalho, F. M., Reis, R. P., Santos, I. C. \& Saraiva, F. H. (2006). Efeito da escala de produção nos resultados econômicos de sistemas de produção de leite na região de Lavras (MG): um estudo multicasos. Boletim da Indústria Animal, 63, 177-188.

Lopes, M. A., Lima, A. L. R., Carvalho, F. M., Reis, R. P., Santos, Í. C. \& Saraiva, F. H. (2004). Controle gerencial e estudo da rentabilidade de sistemas de produção de leite na região de Lavras (MG). Revista Ciência e Agrotecnologia, 28, 883-892.

Lopes, M. A., Santos, G., Resende, M. C., Carvalho, F. M. \& Cardoso, M. G. (2011). Estudo da rentabilidade de sistemas de produção de leite no município de Nazareno, MG. Revista Ciência Animal Brasileira, 12, 58-69.

Lopes, P. F., Reis, R. P. \& Yamaguchi, L. C. T. (2007). Custos e escala de produção na pecuária leiteira: estudo nos principais estados produtores do Brasil. Revista de Economia e Sociologia Rural, 45, 567-590.

Matsunaga, M., Bemelmans, P. \& Toledo, P. (1976). Metodologia de custo de produção utilizada pelo IEA. Agricultura em São Paulo, 23, 123-139.

Oliveira, A. S., Cunha, D. N. F. V., Campos, J. M. S., Vale, S. M. L. R. \& Assis, A. J. (2007). Identificação e quantificação de indicadoresreferência de sistemas de produção de leite. Revista Brasileira de Zootecnia, 36, 507-516.

Oliveira, T. B. A., Figueiredo, R. S., Oliveira, M. \& Nascif, C. (2001). Índices técnicos e rentabilidade da pecuária leiteira. Scientia Agrícola, 58, 687.

Portal Brasil. (2016). Caderneta de poupança. Índices mensais. Disponível em: <portalbrasil.net/poupanca_mensal.htm> Acesso em: 10 jan, 2016.

Silveira, I., Peters, M. D. P., Storch, T., Ziguer, E. A. \& Fischer, V. (2011). Simulação da rentabilidade e viabilidade econômica de um modelo de produção de leite em free-stall. Arquivo Brasileiro de Medicina Veterinária e Zootecnia, 63, 392-398.

Siqueira, K. B., Carneiro, A. V., Almeida, M. F. \& Souza, R. C. N. (2010). O mercado lácteo brasileiro no contexto mundial. Embrapa Gado de Leite, 104, 1-12.

Sousa, M. R. P., Ristow, A. M., Nogueira, E. B., Torres Filho, R. A. \& Cortez, E. M. A. S. (2011). Caracterização de pequenas unidades produtoras de leite na região Centro e Noroeste do estado do Rio de Janeiro. Revista Brasileira de Ciência Veterinária, 18.

Recebido em Janeiro 27, 2016

Aceito em Fevereiro 25, 2016

License information: This is an open-access article distributed under the terms of the Creative Commons Attribution License, which permits unrestricted use, distribution, and reproduction in any medium, provided the original work is properly cited 